\title{
Predictors of influenza among older adults in the emergency department
}

\author{
Po-Po Lam ${ }^{1,2^{*}}$ (D) Brenda L. Coleman ${ }^{1,2}$, Karen Green ${ }^{2,3}$, Jeff Powis ${ }^{4}$, David Richardson ${ }^{5}$, Kevin Katz ${ }^{6}$, \\ Bjug Borgundvaag ${ }^{7}$, Telisha Smith-Gorvie ${ }^{8}$, Jeffrey C. Kwong ${ }^{9}$, Susan J. Bondy ${ }^{1}$ and Allison McGeer ${ }^{1,2,3}$
}

\begin{abstract}
Background: Diagnosis of influenza in older adults may be complicated by atypical presentations or when patients present with complications of an underlying illness. We aimed to identify clinical characteristics and epidemiological factors associated with influenza among community-dwelling adults aged $\geq 60$ years presenting to emergency departments.

Methods: We identified patients with influenza-compatible chief complaints presenting to emergency departments of six acute care hospitals in Ontario, Canada during the 2011/12 and 2012/13 influenza seasons. Clinical characteristics, medical history and demographics were collected by patient interview, chart review and by contacting vaccine providers. Nasopharyngeal swabs were tested for influenza using polymerase chain reaction. We modeled predictors of influenza using multivariable logistic regression models that compared individuals with and without influenza.

Results: Of 1318 participants, 151 (11\%) had influenza (98 A/H3N2, 12 A/H1N1, 4 A [not sub-typed], 37 B). In the multivariable model, clinical symptoms associated with influenza were cough (OR $6.4,95 \% \mathrm{Cl} 3.2,13.0$ ), feverishness and/or triage temperature $\geq 37.2^{\circ} \mathrm{C}(\mathrm{OR} 3.0,95 \% \mathrm{Cl} 2.0,4.7), 2-5$ days from symptom onset to the emergency department visit (OR 2.2, $95 \% \mathrm{Cl}$ 1.5, 3.2), and wheezing (OR 2.1, $95 \% \mathrm{Cl}$ 1.3, 3.3). The effect of cough on influenza increased with older age. Epidemiological factors associated with increased odds for influenza included weeks when $\geq 10 \%$ influenza tests from provincial laboratories were positive (OR 5.1, $95 \% \mathrm{Cl}$ 1.2, 21.7) and exposure to a person with influenza-like illness (OR 1.9, $95 \% \mathrm{Cl}$ 1.3, 2.8). Among participants with influenza, only 47 (31\%) met the U.S. Centers for Disease Control and Prevention criteria for influenza-like illness (temperature $\geq 37.8{ }^{\circ} \mathrm{C}$ and cough and/or sore throat).
\end{abstract}

Conclusions: As in younger adults, cough and feverishness are the two symptoms most predictive of influenza in the elderly. Current influenza-like illness definitions did not adequately capture influenza in older adults.

Keywords: Influenza, Older adults, Elderly, Clinical symptoms

\section{Background}

Accurate and timely diagnosis of influenza is especially important for older adults who are at higher risk for severe complications and for whom influenza vaccines are less effective [1-3]. Laboratory testing with polymerase chain reaction (PCR) is not always available, and most other tests are of relatively low sensitivity and/or

\footnotetext{
* Correspondence: popo.lam@utoronto.ca

${ }^{1}$ Dalla Lana School of Public Health, University of Toronto, Toronto, ON, Canada

${ }^{2}$ Department of Microbiology, Mount Sinai Hospital, Toronto, ON, Canada Full list of author information is available at the end of the article
}

may not yield results in a timely manner [4]. Diagnosis based on signs and symptoms is challenging because influenza symptoms are difficult to distinguish from those of other viral respiratory tract infections (e.g., respiratory syncytial virus, rhinovirus) $[2,5]$, and because influenza may present as a complication of an underlying illness (e.g., exacerbation of chronic obstructive pulmonary disease) rather than as a primary illness [2].

Current evidence on the predictors of influenza in the elderly is limited. Previous studies have identified feverishness, cough, myalgia, pain on respiration, headache, 
sore throat and rigor/chills as clinical symptoms associated with influenza [6-12]. As in younger adults, cough and fever have the highest positive predictive value (PPV) [13]. However, past efforts to identify useful symptom clusters have not been able to determine a combination that discriminate adequately between influenza and other illnesses $[6,8,11,12,14]$. The aim of this study was to identify clinical characteristics and/or epidemiological factors associated with influenza among community-dwelling adults older adults presenting to emergency departments (EDs).

\section{Methods}

\section{Study design and setting}

We prospectively recruited community-dwelling older adults age $\geq 60$ years presenting to the EDs of six acute care hospitals in urban and suburban areas of southcentral Ontario, Canada. These EDs had a median of 175 visits per day (range 160-350). Patients or their substitute decision-makers were approached for consent, nasopharyngeal swabs were collected, and, whenever possible, interviews were conducted while they were in the ED.

\section{Participant enrollment}

Participant enrollment occurred during weeks when the proportion of specimens submitted to Ontario laboratories testing positive for influenza was greater than $5 \%$ for the 2011/12 and 2012/13 seasons (Fig. 1). Study personnel screened and enrolled patients from each site during five, 8-h shifts per week. The shifts were scheduled to sample patients in each ED on all seven days of the week for $24 \mathrm{~h}$ of the day. Study eligible patients who had most recently arrived were approached first by the research assistant to avoid bias introduced by recruiting patients with longer ED stays. Study approval was obtained from all participating hospital research ethics boards (REBs).

Eligible patients had chief complaints of cardiac, respiratory or cerebrovascular events, systemic symptoms (fever, weakness) or altered level of consciousness. Patients with chief complaints related to trauma, mental disorders, post-operation complications and/or urogenital problems were not eligible. For other chief complaints, patients were included if their triage record indicated any of the following symptoms or conditions: respiratory symptoms, triage temperature $\geq 37.5{ }^{\circ} \mathrm{C}$, feverishness, malaise, or general weakness. Residents of long-term care homes or other group settings (e.g., detention centers) and patients who had been enrolled in the study within the previous 14 days were not eligible.

\section{Data collection}

Recruiters interviewed participants to determine symptoms, lifestyle, healthcare use, history of pneumococcal vaccination, and influenza vaccination in the previous five years, medical care/consultation during current illness episode, and Clinical Frailty Scale items [15]. Demographic, medical history, and symptom information were also collected by chart review. The participant's vaccine provider and/or family physician was contacted to obtain influenza vaccination history (for the present and previous season), pneumococcal vaccination history and information on prior visits related to the current episode of illness. Vaccine providers were re-contacted to confirm vaccination information if provider and patient-reported status were discrepant. If the discrepancy could not be resolved, the 'yes' response from either source was accepted. Similarly, for discrepancies between self-reported

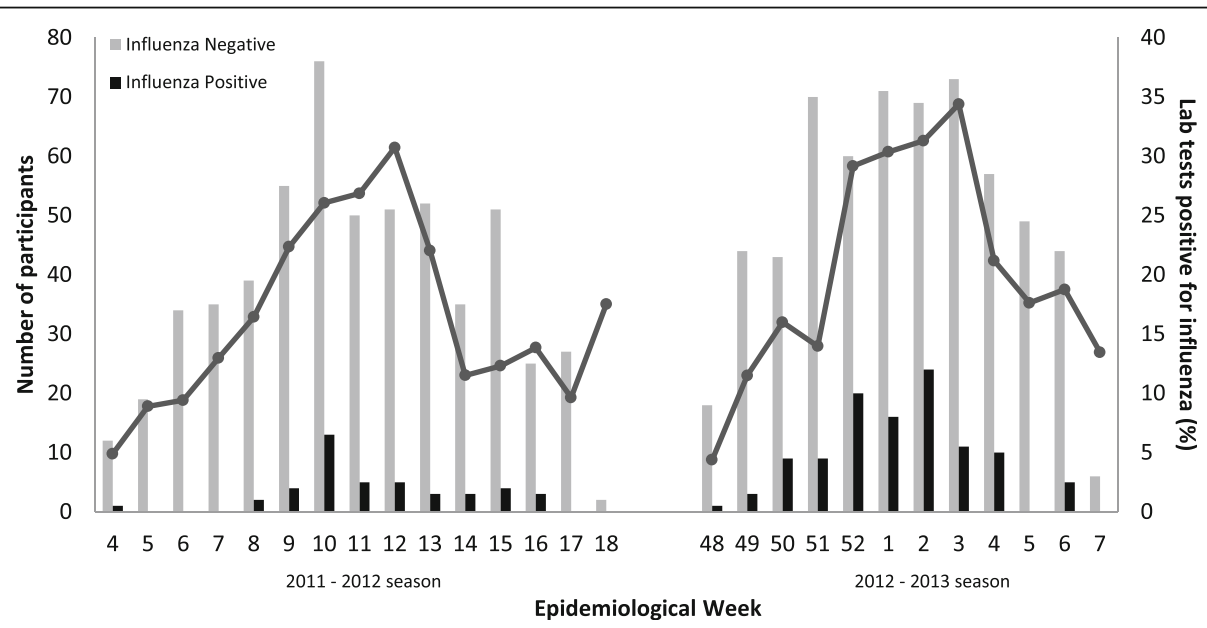

Fig. 1 Participants with and without influenza and the percentage of specimens testing positive for influenza submitted to Ontario laboratories by epidemiological week, 2011-2012 and 2012-2013 seasons 
and chart data for symptoms or medical history, a 'yes' from either source was taken as the final response.

The outcome of interest was laboratory-confirmed influenza infection. A nasopharyngeal swab collected on date of enrolment was tested for influenza by reverse transcription PCR (RT-PCR) at a single study laboratory within $24 \mathrm{~h}$ of collection using bioMérieux easyMAG with RealStar influenza RT-PCR (Altona Diagnostics) in 2011/12 and Simplexa ${ }^{\text {mi }}$ Flu A/B \& RSV Direct RT-PCR (Focus Diagnostics) in 2012/13.

A priori hypothesized predictors of influenza infection were symptoms (cough, fever, acute confusion, sore throat, chills, headache, myalgia, rhinorrhea, wheezing), acute onset of symptoms, influenza vaccination status, children aged $\leq 16$ years in the household, regular contact with children outside the household, exposure to person(s) with influenza-like illness (ILI), recent use of public transit, and the level of influenza activity in the community. We also hypothesized that the association between symptoms and influenza infection would vary across age, presence of underlying illnesses, use of medications for the current episode of illness, deprivation score, and level of frailty.

\section{Predictor definitions}

We chose definitions of predictors to maximize sensitivity and best discriminate between those with and without influenza, with preference given to variables being readily available in ED settings. We assessed self-reported and chart-recorded severity (mild, moderate or severe) of clinical symptoms as well.

The definition of cough was a composite of self-reported and/or chart-recorded data. Participants were recorded as having cough if it was reported during the interview as a symptom prior to the ED visit or if cough was recorded in the medical chart. Fever was defined as self-reported feverishness and/or having a triage-recorded temperature $\geq 37.2{ }^{\circ} \mathrm{C}$ [13]. All participants were asked if they felt feverishness during their current episode of illness. Acute onset of symptoms was defined as being sudden (i.e., woke up feeling miserable or were unable to do regular activities within a few hours) versus gradual development (i.e., symptoms worsened over several days). Time from symptom onset to ED visit was calculated as the number of days between participant-reported day of onset and presentation to the ED.

A participant was defined as having received the influenza vaccine if it was received in the current season and $\geq 14$ days before symptom onset. Participants' chronic conditions were abstracted from medical records and medication history was collected by interview and medical chart review.

Participants were asked if they lived with children ( $\leq 16$ years), and whether they had regular contact $(\geq 4 \mathrm{~h}$ per day and for $\geq 4$ days per week) with other children outside the household. Recent use of public transportation included riding a bus, subway, train, or streetcar within four days of symptom onset. Exposure to a person with an ILI was defined as being within arm's length for $\geq 2$ min to someone the participant recognized as having symptoms of an acute respiratory illness (i.e., had cough, stuffy or runny nose, hoarseness or a sore throat) within 7 days of symptom onset.

Influenza activity in the community was defined as the proportion of specimens positive for influenza submitted to laboratories in south-central Ontario during the past week. Neighborhood-based material and social deprivation indices were determined by matching participants' postal codes with the Canadian Deprivation Index database [16]. The index is divided into 5 quintiles with ' 1 ' being the most privileged and ' 5 ' representing the most deprived.

Ethnicity was self-reported by participants. Frailty was defined as having a score of $\geq 5$ on the Clinical Frailty Scale (maximum 9 points), as scored by a trained research assistant [15], where the participant required at least some assistance with daily activities [17].

\section{Statistical analyses}

We compared the characteristics of individuals having laboratory-confirmed influenza with those who tested negative. Chi-square and Fisher's exact tests were applied to assess differences among group proportions. Medians were compared using Wilcoxon rank-sum tests. Reported $p$-values are two-sided with a value $<0.05$ considered to be statistically significant. No correction was made for multiple testing. Odds ratios (OR) and $95 \%$ confidence intervals $(\mathrm{CI})$ were estimated using logistic regression. Hypothesized predictors significant at $\mathrm{p}<0.2$ in the bivariate analysis were included in the initial logistic regression model [18]. The full model was reduced by sequentially removing predictors that did not significantly improve the fit of the model as determined by the Likelihood Ratio Test [18]. Hosmer-Lemeshow Goodness-ofFit Test and residuals plots were used to assess model fit. Variance inflation factors (VIF >10) were used to assess for collinearity between predictors. No imputation was done for missing data. In the multivariable analysis, participants with missing data on included predictors were excluded. The final model was adjusted for hospital site and had at least ten events per predictor in the model.

Statistical interaction terms, determined a priori, were entered as cross-product terms in a logistic regression model. If significant in the crude analysis, the interaction term was added to the final model and assessed for significance [19]. To interpret effect modification, the odds ratio was calculated for one predictor on the outcome over the levels of another predictor. Vaccine effectiveness was calculated as (1 - OR) X 100. 
To explore potential differences between influenza types (A \& B), we compared each type to influenza-negative participants. We used SAS version 9.3 (SAS Institute, Cary, NC, USA) for all analyses.

\section{Results}

\section{Participants}

Among eligible patients, $64 \%$ were approached by a research assistant and, of those approached, $65 \%$ consented to participate in the study (Fig. 2). In total, 1318 people were enrolled: 606 (46\%) during the 2011/12 and 712 (54 \%) during the 2012/13 influenza seasons. Median participant age was 76.4 years (interquartile range: 68.5-84.2 years), and 656 (50 \%) were female. The majority $(n=868,66 \%)$ had been immunized against influenza in the current season, and $61 \%$ reported one or more underlying chronic diseases. Overall, 151 participants (11\%) tested positive for influenza (98 A/H3N2, $12 \mathrm{~A} / \mathrm{H} 1 \mathrm{~N} 1,4 \mathrm{~A}$ [not sub-typed], $37 \mathrm{~B}$ ). A greater proportion of individuals had influenza during the 2012/13 season $(108 / 712,15 \%)$ compared to the $2011 / 12$ season $(43 / 606,7 \% ; p<0.001)$. The proportion of participants with influenza varied across hospital sites ranging from $6 \%$ to $23 \%$ (median $18 \%$ ).

Participants with influenza and those without influenza did not differ by age, sex, vaccination status, or underlying chronic disease status (Table 1). However, those with influenza were significantly more likely to live in neighborhoods with high material deprivation scores, less likely to be Caucasian, and more likely to have been prescribed antibiotics and used symptom relief medications prior to ED arrival. Only two participants reported the use of an antiviral drug prior to their ED visit.

Among those with influenza, $31 \%$ presented with symptoms that matched the U.S. Centers for Disease Control and Prevention definition for ILI (temperature $\geq 37.8{ }^{\circ} \mathrm{C}$ and cough and/or sore throat) [20], with a specificity of $92 \%$, sensitivity of $31 \%$, PPV of $34 \%$ and negative predictive value (NPV) of $91 \%$. The Public Health Agency of Canada definition for ILI (acute onset of symptoms, fever and cough with sore throat, arthralgia, myalgia or prostration) [21] captured $32 \%$ of the influenza positive participants with a specificity of $91 \%$, sensitivity of $32 \%$, PPV of $31 \%$ and NPV of $91 \%$.

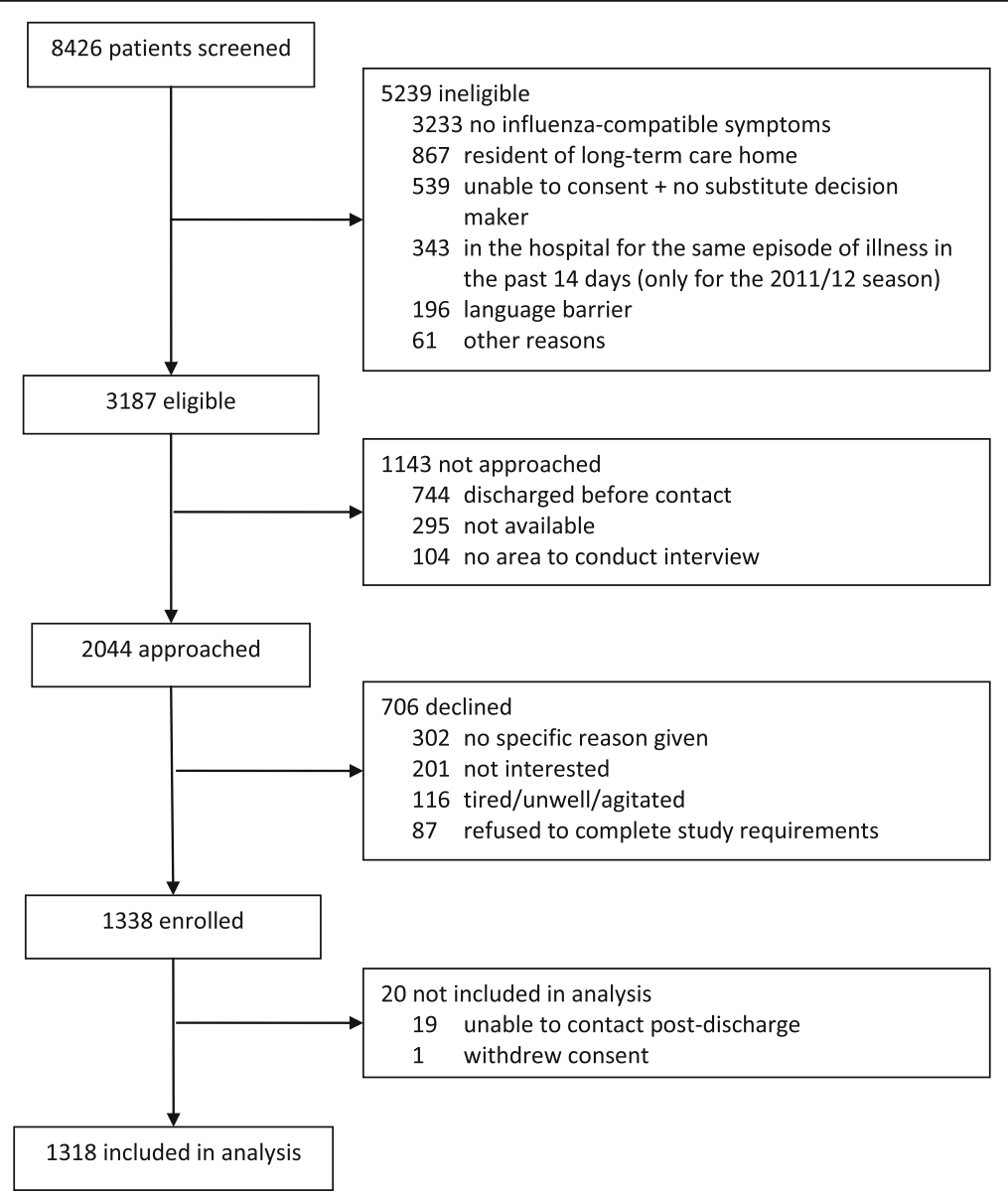

Fig. 2 Flow diagram of participant enrollment 
Table 1 Symptoms and characteristics of participants aged $\geq 60$ years with and without influenza presenting to emergency departments, Ontario, Canada, 2011/12 and 2012/13

\begin{tabular}{|c|c|c|c|}
\hline \multirow{2}{*}{$\begin{array}{l}\text { Participant characteristics/ } \\
\text { symptoms }{ }^{a}\end{array}$} & No Influenza & Influenza & \multirow[t]{2}{*}{$p$-value } \\
\hline & $n=1167(\%)$ & $n=151(\%)$ & \\
\hline Age, median (IQR) & $\begin{array}{l}76.1 \mathrm{yrs} \\
\text { (IQR 68.4-84.1) }\end{array}$ & $\begin{array}{l}77.4 \text { yrs } \\
\text { (IQR 68.9-86.1) }\end{array}$ & .35 \\
\hline Female sex & $578(50)$ & $78(52)$ & .62 \\
\hline $\begin{array}{l}\text { Used public transportation } \\
\leq 4 \text { days before onset }\end{array}$ & $160(14)$ & $23(15)$ & .62 \\
\hline $\begin{array}{l}\text { Children ( } \leq 16 \text { years) } \\
\text { in household }\end{array}$ & $98(8)$ & $17(11)$ & .26 \\
\hline Works with children & $14(1)$ & $5(3)$ & .06 \\
\hline $\begin{array}{l}\text { Exposed to person with ILI } \\
\leq 7 \text { days before onset }\end{array}$ & $275(24)$ & $64(42)$ & $<.001$ \\
\hline $\begin{array}{l}\text { Material deprivation index }{ }^{\dagger}, \\
\text { Median }\end{array}$ & 3 (IQR 1-4) & $3($ IQR 2-5) & .001 \\
\hline White/Caucasian & $895(78)$ & $105(70)$ & .03 \\
\hline $\begin{array}{l}\text { Influenza vaccine, current } \\
\text { season }\end{array}$ & $768(66)$ & $100(68)$ & .75 \\
\hline $\begin{array}{l}\text { Pulmonary /cardiac disease } \\
\text { or immunosuppressed }\end{array}$ & $709(61)$ & $93(62)$ & .84 \\
\hline Frail ( $\geq 5$ on Clinical Frailty Score) & $309(27)$ & $36(24)$ & .35 \\
\hline \multicolumn{4}{|l|}{ Enrolment season: } \\
\hline $2011 / 12$ & $563(93)$ & $43(7)$ & $<.001$ \\
\hline 2012/13 & $604(85)$ & $108(15)$ & \\
\hline $\begin{array}{l}\text { Enrolled week when } \geq 10 \% \\
\text { of influenza tests positive }\end{array}$ & $1057(91)$ & $149(99)$ & $<.001$ \\
\hline \multicolumn{4}{|l|}{ Healthcare use prior to ED arrival } \\
\hline $\begin{array}{l}\text { Prior consultation with health } \\
\text { practitioner }\end{array}$ & $465(40)$ & $66(44)$ & .34 \\
\hline Antibiotics prescribed & $187(16)$ & $40(26)$ & .002 \\
\hline Antipyretics taken & $512(44)$ & $68(45)$ & .79 \\
\hline $\begin{array}{l}\text { Other general symptom } \\
\text { relief medications taken }\end{array}$ & $85(7)$ & $24(16)$ & .001 \\
\hline \multicolumn{4}{|l|}{ Presenting symptoms } \\
\hline Cough (self-report and/or chart) & $649(56)$ & $142(94)$ & $<.001$ \\
\hline Wheezing (chart) & $90(8)$ & $35(23)$ & $<.001$ \\
\hline Sore throat & $244(21)$ & $60(40)$ & $<.001$ \\
\hline Stuffy/runny nose & $353(30)$ & $88(58)$ & $<.001$ \\
\hline Sneezing & $274(24)$ & $50(33)$ & .01 \\
\hline Shortness of breath & $693(59)$ & $98(65)$ & .19 \\
\hline $\begin{array}{l}\text { Any one or more of: sore } \\
\text { throat, runny/stuffy nose } \\
\text { or sneezing }\end{array}$ & $522(45)$ & $111(74)$ & $<.001$ \\
\hline $\begin{array}{l}\text { Feverishness and/or triage } \\
\text { temp } \geq 37.2^{\circ} \mathrm{C}\end{array}$ & $463(40)$ & $115(76)$ & $<.001$ \\
\hline Headache & $419(36)$ & $71(47)$ & .01 \\
\hline Myalgia & $357(31)$ & $71(47)$ & $<.001$ \\
\hline Chills (self-report and/or chart) & $172(15)$ & $57(38)$ & $<.001$ \\
\hline Weakness & $873(75)$ & $125(83)$ & .03 \\
\hline
\end{tabular}

Table 1 Symptoms and characteristics of participants aged $\geq 60$ years with and without influenza presenting to emergency departments, Ontario, Canada, 2011/12 and 2012/13 (Continued)

\begin{tabular}{|c|c|c|c|}
\hline Fatigue & $906(78)$ & $138(91)$ & $<.001$ \\
\hline Loss of appetite & $603(52)$ & $102(68)$ & $<.001$ \\
\hline $\begin{array}{l}\text { Median number of systemic } \\
\text { symptoms (headache, } \\
\text { myalgia, chills, weakness, } \\
\text { fatigue, or loss of appetite) }\end{array}$ & 2 (IQR 2-3) & $3($ IQR 2-4) & $<.001$ \\
\hline $\begin{array}{l}\text { Confused } \\
\text { (self-report and/or chart) }\end{array}$ & $257(22)$ & $46(30)$ & .02 \\
\hline \multicolumn{4}{|l|}{$\begin{array}{l}\text { Time from symptom onset } \\
\text { to ED triage: }\end{array}$} \\
\hline $0-1$ day & $451(39)$ & $25(17)$ & $<.001$ \\
\hline $2-5$ days & $320(27)$ & $81(54)$ & \\
\hline$\geq 6$ days & $393(34)$ & $43(29)$ & \\
\hline Acute onset of symptoms ${ }^{\ddagger}$ & $586(50)$ & $70(47)$ & .33 \\
\hline \multicolumn{4}{|c|}{$\begin{array}{l}I Q R \text { Interquartile range, } E D \text { Emergency department, } I L I \text { influenza-like illness } \\
\text { a'Symptoms and characteristics were based on self-reported data unless } \\
\text { indicated otherwise; The proportion of participants with missing data for any } \\
\text { given predictor did not exceed } 3 \% \\
{ }^{+} \text {Material deprivation index ranks from } 1 \text { (most privileged neighborhood) to } 5 \\
\text { quintiles (most deprived neighborhood) } \\
{ }^{\ddagger} \text { Acute onset was self-defined as symptom development within a few hours ra- } \\
\text { ther than gradual worsening of symptoms } \geq 6 \mathrm{~h}\end{array}$} \\
\hline
\end{tabular}

\section{Predictors of influenza}

Cough as indicated by medical chart review had lower sensitivity (128/151, $85 \%)$ compared with self-report (139/151, $92 \%)$. A composite variable that included self-reported and/or chart-recorded cough achieved the highest sensitivity $(142 / 151,94 \%)$ with $44 \%$ specificity, $18 \%$ PPV and 98 \% NPV. Cough was the most common (94\%) respiratory symptom among those with influenza (Table 1). In the bivariate analysis, all three definitions of coughing were associated with influenza: cough as indicated by chart review (OR 8.9, 95 \% CI 5.6, 14.1), self-reported cough (OR 11.1, $95 \%$ CI 6.1, 20.2) and the composite variable (OR 12.6, $95 \%$ CI 6.4, 24.9).

Participants with influenza had a higher median triage temperature than those without influenza $\left(37.3{ }^{\circ} \mathrm{C}\right.$ versus $\left.36.7{ }^{\circ} \mathrm{C}, p<0.001\right)$. In this sample, $32 \%$ of those with influenza had a triage temperature $\geq 37.8^{\circ} \mathrm{C}$ (specificity $75 \%)$; a lower temperature cut-off of $37.2{ }^{\circ} \mathrm{C}$ captured $55 \%$ of those with influenza (specificity $79 \%$ ). A composite variable of self-reported feverishness and/or measured temperature $\left(\geq 37.2{ }^{\circ} \mathrm{C}\right)$ had the highest sensitivity (76\%), with $60 \%$ specificity, $20 \%$ PPV and $95 \%$ NPV.

Participants with influenza were more likely to report one or more of: wheezing, sore throat, stuffy/runny nose, shortness of breath, or sneezing (Table 1). They were also significantly more likely to report more systemic symptoms (headache, myalgia, chills, weakness, fatigue, or loss of appetite) $(p<0.001)$. 
Individuals with influenza were more likely to present to the ED during weeks with a higher proportion of lab specimens yielding influenza as compared to those without influenza (median $29 \%$ versus $21 \%, p<0.001$, Fig. 1). To guide clinical decision-making, a threshold of $10 \%$ was used to define seasonal influenza activity [22].

In the multivariable model (Table 2), the predictors with the highest ORs for influenza were cough and feverishness. Hospital site did not improve the fit of the regression model (likelihood ratio test $p=0.12$ ) but it was included in the final model to control for possible confounding on the association between predictors and outcome [18].

The OR for the association between cough and influenza infection was modified by age, with the effect of cough being greater in older age groups (Table 3).
Among those who were not frail, confusion (as identified by either patient interview or chart review) was associated with having influenza (adjusted OR 2.0, $95 \% \mathrm{CI}$ $1.2,3.3$; $p$-value for interaction term $=0.004$ ); no association was observed in frail individuals (adjusted OR 0.46, $95 \%$ CI 0.18, 1.1). The effect of influenza vaccination differed across the two study seasons (Breslow-Day test for homogeneity $p=0.01$ ). The estimated vaccine effectiveness (adjusted for predictors and interaction terms in the final model) was $47 \%$ (95\% CI $-8 \%, 74 \%$ ) in 2011/12 and $-51 \%(95 \%$ CI $-154 \%, 11 \%)$ in 2012/13, a year with high $\mathrm{H} 3 \mathrm{~N} 2$ activity.

Almost all influenza B cases (89 \%) were recruited in the first season and the majority of influenza A (91\%) cases were from season two. In an exploratory bivariate analysis, cough, wheezing, feverishness, any upper respiratory

Table 2 Results of final logistic regression model for influenza, Ontario, Canada, 2011/12 and 2012/13

\begin{tabular}{|c|c|c|c|c|}
\hline \multirow[t]{2}{*}{ Predictors $^{a}$} & \multicolumn{2}{|l|}{$\begin{array}{l}\text { Unadjusted OR } \\
(95 \% \mathrm{Cl})\end{array}$} & \multicolumn{2}{|l|}{$\begin{array}{l}\text { Adjusted OR }{ }^{\dagger} \\
(95 \% \mathrm{Cl})\end{array}$} \\
\hline & $n=1313$ & $p$-value & $n=1313$ & $p$-value \\
\hline \multicolumn{5}{|l|}{ Presenting symptoms $^{\ddagger}$} \\
\hline Cough (self-report and/or chart) & $12.4(6.3,24.6)$ & $<.001$ & $6.4(3.2,13.0)$ & $<.001$ \\
\hline Wheeze (chart) & $3.7(2.4,5.7)$ & $<.001$ & $2.1(1.3,3.3)$ & .003 \\
\hline Any one or more of: sore throat, runny/stuffy nose, or sneezing & $3.5(2.4,5.1)$ & $<.001$ & - & \\
\hline Feverishness and/or triage temperature $\geq 37.2^{\circ} \mathrm{C}$ & $4.8(3.2,7.1)$ & $<.001$ & $3.0(2.0,4.7)$ & $<.001$ \\
\hline Number of systemic symptom(s) $)^{z}$ & $1.5(1.3,1.7)$ & $<.001$ & - & \\
\hline Confused (self-report and/or chart) & $1.6(1.1,2.3)$ & .02 & - & \\
\hline Time to ED from symptom onset ( $2-5$ days) & $3.1(2.2,4.5)$ & $<.001$ & $2.2(1.5,3.2)$ & $<.001$ \\
\hline \multicolumn{5}{|l|}{ Medical history } \\
\hline Influenza vaccine, current season & $1.1(0.8,1.6)$ & .59 & - & \\
\hline Antibiotics prescribed/taken prior to visit & $1.9(1.3,2.8)$ & .001 & - & \\
\hline General symptom relief medications prior to visit & $2.4(1.5,4.0)$ & $<.001$ & - & \\
\hline Frail ( $\geq 5$ on Clinical Frailty Score) & $0.78(0.52,1.2)$ & .24 & - & \\
\hline \multicolumn{5}{|l|}{ Other factors } \\
\hline Age, per 10 year increase" & 1. $1(0.9,1.3)$ & .51 & $1.2(1.0,1.5)$ & .03 \\
\hline Children ( $<16$ years) in household & $1.4(0.8,2.4)$ & .21 & - & \\
\hline Recent transportation use & $1.1(0.7,1.8)$ & .58 & - & \\
\hline Exposed to person with ILI $\leq 7$ days before onset & $2.5(1.7,3.5)$ & $<.001$ & $1.9(1.3,2.8)$ & .002 \\
\hline Material deprivation index ${ }^{\mathrm{b}}$ & $1.2(1.1,1.4)$ & .002 & - & \\
\hline Caucasian/White & $0.67(0.46,0.98)$ & .04 & - & \\
\hline Enrolled week when $\geq 10 \%$ of influenza tests positive & $7.7(1.9,31.3)$ & .005 & $5.1(1.2,21.7)$ & .03 \\
\hline
\end{tabular}

OR Odds ratio, $\mathrm{Cl}$ Confidence interval, ED emergency department, $I L I$ influenza-like illness

${ }^{a}$ Participants with missing data on included predictors were excluded from the analysis. The proportion of participants with missing data for any given predictor did not exceed $3 \%$

${ }^{\mathrm{b}}$ Material deprivation index ranks from 1 (most privileged neighborhood) to 5 quintiles (most deprived neighborhood). The index is modeled as a continuous variable with one unit increase equivalent to an increase in quintile

${ }^{\dagger}$ Adjusted model also includes hospital site

${ }^{\ddagger}$ Symptoms were based on self-reported data unless indicated otherwise

${ }^{{ }^{z}}$ Systemic symptoms were headache, myalgia, chills, weakness, fatigue, and loss of appetite. Predictor modeled as a continuous variable with one unit increase per addition of one symptom

"Reference group: $0-1$ days and $>5$ days for time to ED from symptom onset

"Age was modeled as a continuous variable per 10 years increase 
Table 3 Effect of cough on being influenza positive by age, Ontario, Canada, 2011/12 and 2012/13

\begin{tabular}{ll}
\hline Age (years) & $\begin{array}{l}\text { Odds ratio for influenza when } \\
\text { cough is present }(95 \% \mathrm{Cl})^{\mathrm{a}}\end{array}$ \\
\hline 60 & $1.3(0.4,4.6)$ \\
65 & $2.2(0.9,5.7)$ \\
70 & $3.8(1.8,8.2)$ \\
75 & $6.7(3.0,14.9)$ \\
80 & $11.6(4.1,33.2)$ \\
85 & $20.1(4.9,81.9)$ \\
90 & $34.8(5.8,210.6)$ \\
\hline
\end{tabular}

Cl Confidence interval

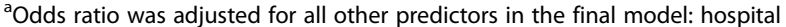
site, wheezing, feverishness, confusion, frailty, time to triage from symptom onset, exposure to person with influenza-like illness, influenza activity in the community, product term of confusion and frailty, and product term of cough and age. Cough was based on chart-review and self-report

symptoms and chills were more prevalent among participants with influenza A as compared to those with influenza B. In our sample, predictors of influenza type was confounded by season of enrolment, as participants in season one were more likely to be hospitalized compared to those recruited in season two (70 \% versus $50 \%, p=0.02)$.

\section{Discussion}

In this prospective cohort study of community-dwelling older adults aged $\geq 60$ years who attended an ED, we found that symptoms of cough, feverishness, and wheezing were independently associated with having influenza. We also identified independent epidemiological predictors: influenza positivity in Ontario laboratory specimens of $\geq 10 \%$, recent exposure to person(s) with ILI, presenting to the ED 2-5 days after symptom onset and advanced age.

Most participants with influenza had cough, which has also been commonly reported in other studies of older adults seeking medical attention $[9,10,12]$. Cough was one of the two strongest predictors of influenza in older adults. Only one other study has identified cough to be a predictor of influenza infection in older adults and with a similar magnitude of effect [6]. In studies that reported a null association [8-11], only participants with ILI were selected, and, as a result, these studies had limited ability to detect the discriminating effect of cough. Cough has also been reported as a clinical predictor of influenza among younger adults and within the same effect size range [13, 23, 24].

In our sample, cough was only associated with influenza among those at advanced ages. The effect of age on the association between cough and influenza is likely related to the combination of the decrease in cough strength with advancing age, and the greater cough stimulus associated with influenza [25].

As seen in previous studies $[6-8,11,12]$, fever (self-reported feverishness and/or measured temperature) has been associated with having influenza among older adults. Measured fever is a known predictor of influenza in younger adults $[13,24]$ but with a higher temperature threshold $\left(\geq 37.8{ }^{\circ} \mathrm{C}\right)$. It is expected that older adults with acute infections present with a lower febrile response as compared with younger adults. Similarly, a previous study on hospitalized patients aged $\geq 65$ years found that the temperature threshold $\geq 37.2{ }^{\circ} \mathrm{C}$ captured $78 \%$ of influenzapositive individuals [26] .

Participants with influenza in our sample were more likely to present to the ED 2-5 days after symptom onset. Similarly, in other studies of community-dwelling adults, patients with influenza most commonly sought healthcare 3-7 days after symptom onset [8, 27]. A recent meta-analysis showed that early treatment (within two days of symptom onset) with neuraminidase inhibitors is associated with decreased mortality risk compared to later treatment [28]. In our sample, $54 \%$ of those with influenza presented too late to receive such early treatment. Improved outcomes for the majority of our participants will require better prevention, a significant change in patient behaviour, and/or treatment options that are effective when started $\geq 48 \mathrm{~h}$ after symptoms onset.

Confusion is a known predictor of acute infection in older adults [29]. In our sample, confusion was only associated with influenza in those who were non-frail, and not among already frail older adults.

Historically, influenza A has been considered to cause more severe disease as compared to influenza B [30]. More recent evidence has shown that influenza B is also capable of causing severe outcomes [30,31] and similar clinical symptoms in adults [32]. We found no distinguishing symptoms between influenza types in older adults. However, it should be noted that the power to identify differences is limited by our relatively small sample size.

Exposure to persons with ILI is a known risk factor for infection as human-to-human transmission occurs readily with influenza viruses [33]. Regular contact with children was not a predictor of influenza in our study. However, $<10 \%$ of our participants had household exposure to any children, and $<3 \%$ had household exposure to children $\leq 5$ years of age. Exposure to children may be a more important factor in populations with different social structures than our study population.

As seen in previous studies, ILI definitions only identified a small proportion of older adults with disease [34]. Syndromic diagnosis of influenza among older adults 
remains a challenge and delays timely identification of disease in a healthcare setting where the virus can be transmitted to staff and other patients.

Strengths of our study include the use of PCR testing for influenza (for high sensitivity), prospective data collection on a broad range of epidemiological and clinical risk factors, and broad inclusion criteria designed to minimize missed influenza patients associated with selection bias.

There are also a number of limitations. Patients with severe disease or more frail individuals may have been more likely to refuse (e.g., decline because of fatigue) or less likely to be approached for inclusion in the study (e.g., patients requiring resuscitation). However, we interviewed family members of those unable to answer whenever possible, and, among those approached, only $6 \%$ declined because they were too tired or unwell. We were not able to consider the impact of vaccination in prior years on presentation, as the data were often missing, and could not be validated.

We are unable to explain differences in rates of influenza between hospital sites; however, none of our study findings changed when the analysis was limited to specific hospitals, or when hospital site was removed from the multivariable model. To reduce interview time for ill patients, we did not use detailed tools for the assessment of confusion, which may have altered our findings. Because there is no appropriate method to account for multiple testing in an exploratory study [35], results are presented without multiplicity adjustments and maybe therefore be prone to Type I error. Our study is limited to two influenza seasons in one geographic area and to individuals presenting to EDs.

In our sample, exposures differed among those who presented to the ED as their first healthcare contact compared to those who consulted with other healthcare providers prior to their ED visit. Patients presenting to EDs may be different in the severity of illness or other characteristics to those presenting at other types of settings (e.g., primary care, walk-in clinics). The exposures to influenza and care-seeking behavior of older adults are likely to vary between populations.

\section{Conclusion}

In summary, in our population of ED patients, predictors for influenza in older adults are similar to predictors previously identified in younger adults, with the exception that "fever" is better defined using a lower triage temperature threshold. Current clinical definitions of influenza do not adequately capture influenza in older adults. Future research should focus on determining a useful clinical decision rule for influenza in older adults.

\section{Abbreviations}

Cl: Confidence interval; ED: Emergency department; ILI: Influenza-like illness; IQR: Interquartile range; NPV: Negative predictive value; OR: Odds ratio; PCR: Polymerase chain reaction; PPV: Positive predictive value; REB: Research ethics board; RT-PCR: Reverse transcription polymerase chain reaction; VIF: Variance inflation factor

\section{Acknowledgements}

We would like to thank the emergency department patients for their participation in this study, hospital staff for their collaboration, and the research assistants for their work on this project. PL was supported by a doctoral scholarship from the Canadian Institutes of Health Research.

\section{Funding}

This research was sponsored in part by an unrestricted grant from Hoffman-La Roche Ltd Roche. The funder had no role in the design, conduct, analysis or interpretation of study data.

\section{Availability of data and materials}

The dataset for this study contains personal health information and cannot be shared with individuals outside the study team, as per REB rules.

\section{Authors' contributions}

$\mathrm{PL}, \mathrm{BLC}, \mathrm{KG}, \mathrm{KJ}, \mathrm{SJB}$, and $\mathrm{AM}$ contributed to the conception and design of the study, analysis and interpretation of the data and drafting of the manuscript. JP, DR, KK, BB, and TSG contributed to the design of the study and reviewed the manuscript. All authors read and approved the final manuscript.

\section{Competing interests}

The authors declare that they have no competing interests.

\section{Consent for publication}

The manuscript does not contain any individual participant's data.

\section{Ethics approval and consent to participate}

The study was approved by all participating hospital research ethics boards (REBs), which included: the Mount Sinai Hospital REB, the North York General Hospital REB, the Toronto East General Hospital REB, the University Health Network REB, and the William Osler Health System REB. Written informed consent was obtained from all participants or by their legally authorized representative when the participant was unable to give consent due to illness.

\section{Author details}

'Dalla Lana School of Public Health, University of Toronto, Toronto, ON, Canada. ${ }^{2}$ Department of Microbiology, Mount Sinai Hospital, Toronto, ON, Canada. ${ }^{3}$ Toronto Invasive Bacterial Diseases Network, Toronto, ON, Canada. ${ }^{4}$ Toronto East General Hospital, Toronto, ON, Canada. ${ }^{5}$ William Osler Health System, Toronto, ON, Canada. ${ }^{6}$ North York General Hospital, Toronto, ON, Canada. ${ }^{7}$ Schwartz/Reisman Emergency Medicine Institute, Mount Sinai Hospital, Toronto, ON, Canada. ${ }^{8}$ Department of Emergency Medicine, University Health Network, Toronto, ON, Canada. ${ }^{9}$ Institute for Clinical Evaluative Sciences, Toronto, ON, Canada.

Received: 8 March 2016 Accepted: 25 October 2016

Published online: 28 October 2016

\section{References}

1. Schanzer DL, Langley JM, Tam TW. Role of influenza and other respiratory viruses in admissions of adults to Canadian hospitals. Influenza Other Respi Viruses. 2008;2(1):1-8.

2. Fleming DM, Elliot AJ. The impact of influenza on the health and health care utilisation of elderly people. Vaccine. 2005;23 Suppl 1:S1-9.

3. Lang PO, Mendes A, Socquet J, Assir N, Govind S, Aspinall R. Effectiveness of influenza vaccine in aging and older adults: comprehensive analysis of the evidence. Clin Interv Aging. 2012;7:55-64.

4. Fleming DM. Influenza diagnosis and treatment: a view from clinical practice. Philos Trans R Soc Lond B Biol Sci. 2001;356(1416):1933-43. 
5. Talbot HK, Falsey AR. The diagnosis of viral respiratory disease in older adults. Clin Infect Dis. 2010;50(5):747-51.

6. Govaert TM, Dinant GJ, Aretz K, Knottnerus JA. The predictive value of influenza symptomatology in elderly people. Fam Pract. 1998;15(1):16-22.

7. Graat JM, Schouten EG, Heijnen MLA, Kok FJ, Pallast EGM, De Greeff SC, et al. A prospective, community-based study on virologic assessment among elderly people with and without symptoms of acute respiratory infection. J Clin Epidemiol. 2003;56(12):1218-23.

8. Nicholson KG, Kent J, Hammersley V, Cancio E. Acute viral infections of upper respiratory tract in elderly people living in the community: comparative, prospective, population based study of disease burden. BMJ. 1997;315(7115):1060-4

9. Pitigoi D, Lupulescu E, Alexandrescu V, Baetel A, Sbarcea C, lonita E, et al. Efficiency of seasonal influenza vaccine in persons older than 65 in Romania. Pilot case-control study I-MOVE, 2008-2009. Bacteriol Virusol Parazitol Epidemiol. 2009;54(2):109-16.

10. Savulescu C, Valenciano M, de Mateo S, Larrauri A. Estimating the influenza vaccine effectiveness in elderly on a yearly basis using the Spanish influenza surveillance network-Pilot case-control studies using different control groups, 2008-2009 season, Spain. Vaccine. 2010;28(16):2903-7.

11. Shahid Z, Kleppinger A, Gentleman B, Falsey AR, McElhaney JE. Clinical and immunologic predictors of influenza illness among vaccinated older adults. Vaccine. 2010;28(38):6145-51.

12. Walsh EE, Cox C, Falsey AR. Clinical features of influenza A virus infection in older hospitalized persons. J Am Geriatr Soc. 2002;50(9):1498-503.

13. Monto AS, Gravenstein S, Elliott M, Colopy M, Schweinle J. Clinical signs and symptoms predicting influenza infection. Arch Intern Med. 2000;160(21):3243-7.

14. Michiels B, Thomas I, Van Royen P, Coenen S. Clinical prediction rules combining signs, symptoms and epidemiological context to distinguish influenza from influenza-like illnesses in primary care: a cross sectional study. BMC Fam Pract. 2011;12(1):4

15. Rockwood K, Song X, MacKnight C, Bergman H, Hogan DB, McDowell I, et al. A global clinical measure of fitness and frailty in elderly people. CMAJ. 2005;173(5):489-95.

16. Pampalon R, Hamel D, Gamache P, Philibert MD, Raymond G, Simpson A. An area-based material and social deprivation index for public health in Quebec and Canada. Can J Public Health. 2012;103(8 Suppl 2):S17-22.

17. Hubbard RE, Andrew MK, Fallah N, Rockwood K. Comparison of the prognostic importance of diagnosed diabetes, co-morbidity and frailty in older people. Diabet Med. 2010;27(5):603-6.

18. Vittinghoff E, Glidden DV, Shiboski SC, McCulloch CE. Chapter 10 - Predictor Selection. Regression Methods in Biostatistics Linear, Logistic, Survival, and Repeated Measures Models. 2nd ed. New York: Springer Science; 2012. p. 395-429.

19. Hosmer DW, Lemeshow S, Sturdivant R. Model-building strategies and methods for logistic regression. Applied logistic regression. 3rd ed. United States: Wiley; 2013. p. 448-55.

20. Overview of influenza surveillance in the United States. Centers for Disease Control and Prevention; 2013. http://www.cdc.gov/flu/pdf/weekly/overview. pdf. Accessed June 24 2014;

21. FluWatch: Definitions for the 2012-2013 season. Public Health Agency of Canada; 2012. http://www.phac-aspc.gc.ca/fluwatch/12-13/def12-13-eng. php. Accessed June 17 2013;

22. Kwong JC, Stukel TA, Lim J, McGeer AJ, Upshur RE, Johansen H, et al. The effect of universal influenza immunization on mortality and health care use. PLoS Med. 2008:5(10):e211.

23. Shah SC, Rumoro DP, Hallock MM, Trenholme GM, Gibbs GS, Silva JC, et al. Clinical predictors for laboratory-confirmed influenza infections: exploring case definitions for influenza-like illness. Infect Control Hosp Epidemiol. 2015;36(3):241-8.

24. Carrat F, Tachet A, Rouzioux C, Housset B, Valleron AJ. Evaluation of clinical case definitions of influenza: detailed investigation of patients during the 1995-1996 epidemic in France. Clin Infect Dis. 1999;28(2):283-90.

25. Lowery EM, Brubaker AL, Kuhlmann E, Kovacs EJ. The aging lung. Clin Interv Aging. 2013:8:1489-96

26. Falsey AR, Baran A, Walsh EE. Should clinical case definitions of influenza in hospitalized older adults include fever? Influenza Other Respir Viruses. 2015;9 Suppl 1:23-9
27. Biggerstaff M, Jhung MA, Reed C, Fry AM, Balluz L, Finelli L. Influenza-like Illness, the Time to Seek Healthcare, and Influenza Antiviral Receipt During the 2010-2011 Influenza Season—United States. J Infect Dis. 2014;210(4):535-44.

28. Muthuri SG, Venkatesan S, Myles PR, Leonardi-Bee J, Al Khuwaitir TS, Al Mamun $A$, et al. Effectiveness of neuraminidase inhibitors in reducing mortality in patients admitted to hospital with influenza A H1N1pdm09 virus infection: a meta-analysis of individual participant data. Lancet Respir Med. 2014;2(5):395-404.

29. Inouye SK, Westendorp RG, Saczynski JS. Delirium in elderly people. Lancet. 2014;383(9920):911-22

30. Paddock CD, Liu L, Denison AM, Bartlett JH, Holman RC, Deleon-Carnes M, et al. Myocardial injury and bacterial pneumonia contribute to the pathogenesis of fatal influenza B virus infection. J Infect Dis. 2012;205(6):895-905.

31. Lee N, Choi KW, Chan PKS, Hui DSC, Lui GCY, Wong BCK, et al. Outcomes of adults hospitalised with severe influenza. Thorax. 2010;65(6):510-5.

32. Irving SA, Patel DC, Kieke BA, Donahue JG, Vandermause MF, Shay DK, et al. Comparison of clinical features and outcomes of medically attended influenza $A$ and influenza $B$ in a defined population over four seasons: 2004-2005 through 2007-2008. Influenza Other Respir Viruses. 2012;6(1):37-43.

33. Belser JA, Maines TR, Tumpey TM, Katz JM. Influenza A virus transmission: contributing factors and clinical implications. Expert Rev Mol Med. 2010;12:e39.

34. Yoshikawa TT, Stefan G, Davidson HE. Current Strategies for Management of Influenza in the Elderly Population. Clin Infect Dis. 2002;35(6):729-37.

35. Bender R, Lange S. Adjusting for multiple testing-when and how? J Clin Epidemiol. 2001;54(4):343-9.

\section{Submit your next manuscript to BioMed Central and we will help you at every step:}

- We accept pre-submission inquiries

- Our selector tool helps you to find the most relevant journal

- We provide round the clock customer support

- Convenient online submission

- Thorough peer review

- Inclusion in PubMed and all major indexing services

- Maximum visibility for your research

Submit your manuscript at www.biomedcentral.com/submit
) Biomed Central 